J. Dairy Sci. 92:739-748

doi:10.3168/jds.2008-1325

(c) American Dairy Science Association, 2009.

\title{
Assessment of Poisson, logit, and linear models for genetic analysis of clinical mastitis in Norwegian Red cows
}

\author{
A. I. Vazquez, ${ }^{\star 1}$ D. Gianola, ${ }^{\star} \dagger$ D. Bates,‡ K. A. Weigel, ${ }^{\star}$ and B. Heringstad§ \\ *Department of Dairy Science, \\ †Department of Animal Sciences, and \\ ‡Department of Statistics, University of Wisconsin, Madison 53706 \\ $\S$ Department of Animal and Aquacultural Sciences, Norwegian University of Life Sciences, N-1432 Ås, Norway
}

\begin{abstract}
Clinical mastitis is typically coded as presence/absence during some period of exposure, and records are analyzed with linear or binary data models. Because presence includes cows with multiple episodes, there is loss of information when a count is treated as a binary response. The Poisson model is designed for counting random variables, and although it is used extensively in epidemiology of mastitis, it has rarely been used for studying the genetics of mastitis. Many models have been proposed for genetic analysis of mastitis, but they have not been formally compared. The main goal of this study was to compare linear (Gaussian), Bernoulli (with logit link), and Poisson models for the purpose of genetic evaluation of sires for mastitis in dairy cattle. The response variables were clinical mastitis $(\mathrm{CM} ; 0,1)$ and number of CM cases (NCM; $0,1,2, \ldots$ ). Data consisted of records on 36,178 first-lactation daughters of 245 Norwegian Red sires distributed over 5,286 herds. Predictive ability of models was assessed via a 3-fold cross-validation using mean squared error of prediction (MSEP) as the end-point. Between-sire variance estimates for NCM were 0.065 in Poisson and 0.007 in the linear model. For CM the between-sire variance was 0.093 in logit and 0.003 in the linear model. The ratio between herd and sire variances for the models with NCM response was 4.6 and 3.5 for Poisson and linear, respectively, and for model for $\mathrm{CM}$ was 3.7 in both logit and linear models. The MSEP for all cows was similar. However, within healthy animals, MSEP was 0.085 (Poisson), 0.090 (linear for NCM), 0.053 (logit), and 0.056 (linear for $\mathrm{CM}$ ). For mastitic animals the MSEP values were 1.206 (Poisson), 1.185 (linear for NCM response), 1.333 (logit), and 1.319 (linear for CM response). The models for count variables had a better performance when predicting diseased animals and also
\end{abstract}

Received May 2, 2008.

Accepted August 25, 2008.

${ }^{1}$ Corresponding author: aivazquez@wisc.edu had a similar performance between them. Logit and linear models for CM had better predictive ability for healthy cows and had a similar performance between them.

Key words: mastitis, dairy cattle, Poisson, model comparison

\section{INTRODUCTION}

Mastitis is the most important disease in dairy cattle (National Mastitis Council, 2004). As for many diseases, there are several ways in which the trait can be defined and recorded. In genetic evaluations, clinical mastitis $(\mathbf{C M})$ is often treated as a binary trait, scored as 1 if the cow had at least one treatment within a defined time interval and 0 otherwise. Alternatively, because many events may take place within an exposure period, the number of cases of mastitis (NCM) can be considered as the trait of interest. From a longitudinal perspective, the trait can be defined as the time elapsed for an event to occur, or, as a sequence of binary traits, each of which indicates whether or not an event took place within a subperiod.

Genetic analysis of CM is usually done using a Gaussian (e.g., Rupp and Boichard, 1999) or Bernoulli model (e.g., Heringstad et al., 2001; De Haas et al., 2002). In epidemiology, NCM has been studied using Poisson models (e.g., Schukken et al., 1990; Gasqui et al., 2000); alternatively, if overdispersion is suspected, a zero-inflated-Poisson model may be considered for NCM (Rodrigues-Motta et al., 2007). For longitudinal data, some of the models that have been considered are longitudinal probit (e.g., Rekaya et al., 1998), multivariate probit (Heringstad et al., 2004), and survival (e.g., Sæbø et al., 2005).

Although many models have been proposed and used, these alternatives have not been formally compared. The main goal of this research is to formally compare some of the most commonly used models for genetic analysis of CM and NCM. In particular, the Bernoulli and Gaussian models were considered for the analysis 
of CM, and the Poisson and Gaussian models were considered for the analysis of NCM. Models were compared based on their predictive ability in cross-validation, and parameter estimates and predictions (of sire and herd effects) from each of these models are discussed.

\section{MATERIALS AND METHODS}

\section{Data}

Data consisted of counts of veterinary treatments for $\mathrm{CM}$ during the first lactation for 36,178 cows. These cows were daughters of 245 Norwegian Red sires that had their first progeny test in 1990, 1991, or 1992 and were from 5,286 herds. A 7-generation pedigree file of sires, with a total of 427 males, was used to compute additive relationships. For each cow, mastitis records from $30 \mathrm{~d}$ before first calving to culling, $300 \mathrm{~d}$ after first calving, or second calving, whichever occurred first, were included. Response variables studied were NCM, a count variable with 4 outcomes $(0,1,2$, and 3 or more) and $\mathrm{CM}$, a binary variable taking values 0 (absence) or 1 (presence), during first lactation. This data set has been previously used for variance components estimation (Heringstad et al., 2003; Rodrigues-Motta et al., 2007) and was considered a suitable choice for model comparison.

\section{Statistical Models}

All the models used belong to the class of generalized linear mixed models (GLMM); this class applies to members of the exponential family distributions and is based on finding a relationship (link function) between the expected value of a random variable and a linear function of explanatory variables (McCullagh and Nelder, 1989; Agresti, 2002). The GLMM can be used for predicting genetic values given the genetic variancecovariance structure. Predictions can be obtained by introducing genetic relationships between individuals into the model (Henderson, 1976; Quaas and Pollak, 1980). Alternatively, this can be accomplished by transforming pertinent random effects, such that these become uncorrelated (Harville and Callanan, 1989).

The following linear predictor was common to all models:

$$
\eta_{i j k l m}=\beta_{0}+A g_{i}+Y S_{j}+h_{k}+s_{l}+b \log \left(D I M_{m}\right),
$$

where $\eta_{i j k l m}$ is a function of the expected NCM or CM in first lactation for cow $m ; \beta_{0}$ is an intercept; $A g_{i}$ is the fixed effect of age at calving $(i=1,2,3) ; Y S_{j}$ is the fixed effect of year-season $(j=1,2, \ldots, 12) ; \quad h_{k}$ is a random effect for herd $(k=1,2, \ldots, 5,286)$; and $s_{l}$ is a random effect for sire of cow $m(l=1,2, \ldots, 245) ; D I M_{m}$ is the number of days in milk the cow had in her first lactation; $b$ is a fixed regression coefficient. Age at calving was classified into 3 classes: $<24,24$ to 28 , and $>28$ mo. Twelve year-season of calving groups were formed, resulting from combining $3 \mathrm{yr}(1990,1991$, and 1992) with 4 seasons (February to April, May to July, August to October, and November to January). The following distribution was assumed for the vector of random effects:

$$
\left(\begin{array}{l}
\mathbf{s} \\
\mathbf{h}
\end{array}\right) \sim \mathbf{N}\left[\mathbf{0},\left(\begin{array}{cc}
\mathbf{A} \boldsymbol{\sigma}_{s}^{2} & 0 \\
0 & \mathbf{I} \boldsymbol{\sigma}_{h}^{2}
\end{array}\right)\right],
$$

where $\mathbf{s}=\left\{s_{l}\right\}$ is a vector of sire effects; $\mathbf{h}=\left\{h_{k}\right\}$ is a vector of herd effects; $\sigma_{s}^{2}$ and $\sigma_{h}^{2}$ are the between-sire and between-herd variances, respectively; A represents the matrix of additive relationships between sires; and I is an identity matrix $(5,286 \times 5,286)$.

At the likelihood conditional independence was assumed, i.e., the conditional probability of the response is

$$
P(\mathbf{y} \mid \boldsymbol{\beta}, \mathbf{h}, \mathbf{s})=\prod_{i} \prod_{j} \prod_{k} \prod_{l} \prod_{m} P\left(y_{i j k l m} \mid \boldsymbol{\beta}, \mathbf{h}, \mathbf{s}\right),
$$

where $\boldsymbol{\beta}=\left\{A g_{1}, A g_{2}, A g_{3}, Y S_{1}, \ldots Y S_{12}, b\right\} ; \quad y_{i j k l m}$ is the response (NCM or $\mathrm{CM})$; and $P\left(y_{i j k l m} \mid \boldsymbol{\beta}, \mathbf{h}, \mathbf{s}\right)$ changed across models.

Poisson Model. A Poisson mixed effects model was fitted for NCM. In this model,

$$
P\left(N C M_{i j k l m} \mid \boldsymbol{\beta}, \mathbf{h}, \mathbf{s}\right)=\operatorname{Poisson}\left(\lambda_{i j k l m}\right),
$$

where $\lambda_{i j k l m}$ is a record-specific Poisson parameter satisfying

$$
\begin{aligned}
& E\left(N C M_{i j k l m} \mid \boldsymbol{\beta}, \mathbf{h}, \mathbf{s}\right)=\lambda_{i j k l m}=\exp \left(\boldsymbol{\eta}_{i j k l m}\right) \\
& =\exp \left[\beta_{0}+A g_{i}+Y S_{j}+h_{k}+s_{l}+b \log \left(D I M_{i j k l m}\right)\right] \\
& \text { or } \eta_{i j k l m}=\log \left(\boldsymbol{\lambda}_{i j k l}\right)=\log \left(\lambda_{i j k l}^{*}\right)+b \log \left(D I M_{i j k l m}\right) .
\end{aligned}
$$

Here, $\lambda_{i j k l}^{*}=\exp \left(\beta_{0}+A g_{i}+Y S_{j}+h_{k}+s_{l}\right)$ is the expected number of cases per day at risk. Including days 
in milk as a covariate accounts for variation due to length of exposure, but it was assumed that risk was constant across the entire period of lactation.

Logit Model. The following logistic regression model was fitted

$$
P\left(C M_{i j k l m} \mid \mathbf{\beta}, \mathbf{h}, \mathbf{s}\right)=\operatorname{Bernoulli}\left(\vartheta_{i j k l m}\right),
$$

where $\vartheta_{i j k l m}$ is a record-specific Bernoulli parameter, and

$$
E(C M \mid \boldsymbol{\beta}, \mathbf{h}, \mathbf{s})=\vartheta_{i j k l m}=\frac{\exp \left(\eta_{i j k l m}\right)}{1+\exp \left(\eta_{i j k l m}\right)}
$$

Linear Model. In this model the likelihood function was

$$
P\left(y_{i j k l m} \mid \boldsymbol{\beta}, \mathbf{h}, \mathbf{s}\right)=N\left(\eta_{i j k l m}, \sigma_{\varepsilon}^{2}\right)
$$

where the response $y_{i j k l m}$ equals to either $C M_{i j k l m}$ or $N C M_{i j k l m}$. Most of the literature uses linear models with CM as response (e.g., Rupp and Boichard, 1999). Here,

$$
E(C M \mid \boldsymbol{\beta}, \mathbf{h}, \mathbf{s})=\eta_{i j k l m}, \text { and } E(N C M \mid \boldsymbol{\beta}, \mathbf{h}, \mathbf{s})=\eta_{i j k l m}
$$

\section{Heritability Estimates}

For linear sire models, heritability is defined as

$$
h^{2}=\frac{4 \sigma_{s}^{2}}{\sigma_{s}^{2}+\sigma_{h}^{2}+\sigma_{\varepsilon}^{2}} \text {. }
$$

For nonlinear models, the variance components do not show the same additive partition.

For the Poisson model, Foulley et al. (1987) suggested a heritability approximation in the $\eta$-scale (log scale), which in this case would be

$$
h_{\eta}^{2}=\frac{4 \sigma_{s}^{2}}{\sigma_{s}^{2}+\sigma_{h}^{2}+\lambda^{-1}},
$$

where $\lambda$ is the "average" Poisson parameter for the entire risk period. In this study, $\lambda_{i j k l m}$ was a subject- specific parameter and was evaluated at the mean and median of the population.

In the logistic regression, assume a logistic random variable $\theta_{i j k l m}$, with cumulative density function,

$$
F\left(\theta_{i j k l m} \mid \mu, \alpha\right)=\frac{1}{1+\exp \left[-\frac{(\theta-\mu)}{\alpha}\right]}
$$

with $E(\theta)=\mu$ and $\operatorname{var}(\theta)=\frac{\pi^{2}}{3} \alpha^{2}$.

$$
\text { Let } e=\left(\frac{\theta-\mu}{\alpha}\right) \text {, then } E(e)=0 \text { and } \operatorname{var}(e)=\frac{\pi^{2}}{3} \text {. }
$$

Suppose now that in a logistic scale, latent variable "phenotypes" are formed as $y=s+e$, where $s \sim N\left(0, \sigma_{s}^{2}\right)$ is a sire effect. Then, the variance of the phenotypes would be $\sigma_{y}^{2}=\sigma_{s}^{2}+\frac{\pi^{2}}{3}$. In a "sire" model, the additive variance is estimated as $4 \sigma_{s}^{2}$, then heritability would be

$$
h^{2}=\frac{4 \sigma_{s}^{2}}{\sigma_{s}^{2}+\pi^{2} / 3} \text {. }
$$

\section{Implementation}

A modified version of the lme4 package of $\mathrm{R}$ ( $\mathrm{R}$ Development Core Team, 2008), was used to fit the models. The lme4 package (Bates and Sarkar, 2005) allows fitting GLMM; however, the lmer procedure (in the lme4 package) assumes independence across levels of the grouping factor. This is not correct for sires, where the assumption is $N\left(\mathbf{0}, \mathbf{A} \sigma_{s}^{2}\right)$. Changes were made in lmer to allow the inclusion of an additive relationship matrix between sires. Following Harville and Callanan (1989), random effects were transformed using the Cholesky factor of $\mathbf{A}, \mathbf{s}^{*}=\mathbf{L}^{-1} \mathbf{s}$, where $\mathbf{L}$ is the Cholesky decomposition of $\mathbf{A}$ and $\mathbf{s}^{*} \sim N\left(\mathbf{0}, \mathbf{I} \sigma_{s}^{2}\right)$. An $\mathrm{R}$ function was created to compute the Cholesky decomposition of the relationship matrix from the pedigree (Vazquez, 2007). This reparameterization allowed using lmer and may have computational advantages (i.e., achieving a faster convergence in iterative algorithms). A transformed and more current version of this implementation is publicly available (http:// pedigreemm.r-forge.r-project.org/). The lmer procedure 
for GLMM uses an approximation to the log-likelihood for inferring fixed effects and variance components and was set to use a penalized quasi-likelihood method for obtaining predictions of random effects.

\section{Prediction of Random Effects}

The conditional expectation is the best predictor in a mean squared error sense (Henderson, 1973; Bulmer, 1980). In linear models the best predictor of random effects given the dispersion parameters has closed form (Henderson, 1975). Typically, animal breeders replace the unknown dispersion parameters by restricted maximum likelihood estimates (Gianola and Fernando, 1986). However, in Poisson or logit models the counterparts of the BLUP cannot be written in closed form. Procedures for predicting the counterpart of the BLUP in a Poisson model (Foulley et al., 1987) and probit and logit models (Gianola and Foulley, 1983) have been described in animal breeding. The lmer procedure implements full maximum likelihood estimation of the parameters of the model (i.e., fixed effects and variance components) using Laplacian approximation to the marginal likelihood. Subsequently, predictions of random effects are obtained as the mode of the conditional distribution of the random effects given the parameters evaluated at the maximum likelihood estimates (Doran et al., 2007).

\section{Predictive Ability}

A 3-fold cross-validation was carried out to assess the predictive ability of the 3 models. Similar cross-validation procedures have been used successfully in animal breeding (Caraviello et al., 2004). A 3-fold cross-validation suited to the situation of this study with a relatively large data set was carried out. This cross-validation is not as computationally demanding as a leaveone-out could be and makes a complete use of the data (versus only having one training and one testing subset). Data were split randomly into 3 disjoint folds, containing $n_{1}, n_{2}$, and $n_{3}$ observations. The loss function was the mean squared error of prediction (MSEP), defined as

$$
M S E P=\frac{1}{n} \sum_{f=1}^{3} \sum_{(i j k l m) \in F_{f}}\left[\hat{E}\left(y_{i j k l m} \mid \boldsymbol{\beta}^{(-f)}, \mathbf{h}^{(-f)}, \mathbf{s}^{(-f)}\right)-y_{i j k l m}\right]^{2}
$$

where $y_{i j k l m}$ is a record of an observation in the $f^{\text {th }}$ fold; $\hat{E}\left(y_{i j k l m} \mid \cdot\right)$ represents the prediction of a record using estimates based on data from the other 2 folds; and $n$ is the number of observations for which the MSEP is being computed. Above, $\boldsymbol{\beta}^{(-f)}, \mathbf{h}^{(-f)}$, and $\mathbf{s}^{(-f)}$ denote the fixed and random effects of sires and herds, respectively, from a partition without the $f^{\text {th }}$ subset in it. The predicted value $\hat{E}\left(y_{i j k l m} \mid \boldsymbol{\beta}^{(-f)}, \mathbf{h}^{(-f)}, \mathbf{s}^{(-f)}\right)$ depends on the model used. In the Poisson model $\hat{E}\left(y_{i j k l m} \mid \cdot\right)=\exp \left(\hat{\eta}_{i j k l m}^{(-f)}\right)=\hat{\lambda}_{i j k l m}^{(-f)}$, in the logit model $\hat{E}\left(y_{i j k l m} \mid \cdot\right)=\frac{\exp \left(\hat{\eta}_{i j k l m}^{(-f)}\right)}{1+\exp \left(\hat{\eta}_{i j k l m}^{(-f)}\right)}=\hat{\vartheta}_{i j k l m}^{(-f)}$, and in the linear model $\hat{E}\left(y_{i j k l m} \mid \cdot\right)=\hat{\eta}_{i j k l m}^{(-f)}$.

Comparisons based on mean squared error (MSE) are valid if all models have the same response variable. To illustrate this, let $y_{i}=\mu+\varepsilon_{i}(i=1, \ldots, \mathrm{n})$ (model 1) with $\varepsilon_{i} \sim N\left(0, \sigma_{\varepsilon}^{2}\right)$, residuals assumed independent and identically distributed. The maximum likelihood estimator (MLE) of $\mu$, is $\bar{y}=\frac{1}{n} \sum y_{i}$, and the $M S E=\frac{1}{n} \sum\left(y_{i}-\bar{y}\right)^{2}=s^{2}$. If $y_{i}$ is transformed into $2 y_{i}=2 \mu+2 \varepsilon_{i}($ model 2$)$, in this model the MLE of $\mu$, is $2 \bar{y}=\frac{2}{n} \sum y_{i}, \quad$ and the $M S E=\frac{1}{n} \sum\left(2 y_{i}-2 \bar{y}\right)^{2}=\tilde{s}^{2}=4 s^{2}$. Thus, however, the MSE of model 1 is 4 times smaller than the MSE of model 2. This also extends to the MSEP. Models were evaluated using $N C M_{i j k l m}$ as response for the MSEP.

\section{RESULTS AND DISCUSSION}

\section{Descriptive Statistics}

The mean and variance of NCM were 0.295 and 0.353 , respectively (1.20 variance to mean ratio). About $77 \%$ of the cows did not have any case of mastitis, $18 \%$ had 1 case, $4 \%$ had 2 cases, and $1 \%$ had 3 or more cases during first lactation. Collapsing the counts 1, 2, and 3 or more into a single category reduces variability. The mean and variance of CM were 0.233 and 0.179 , respectively ( 0.77 variance to mean ratio).

\section{Parameter Estimates}

Age at calving, year-season of calving and the regression on $\log (\mathrm{DIM})$ did not have significant effects (F-test). As the focus of this study is evaluation of predictive ability, all fixed effects including $\log (\mathrm{DIM})$ were 
Table 1. Estimates of variance components for clinical mastitis (CM) and number of CM cases (NCM) from Poisson, linear, and logit models

\begin{tabular}{lcccc}
\hline Variance component & Poisson & Linear for NCM & Linear for CM & Logit \\
\hline Sires & 0.0647 & 0.0065 & 0.0030 & 0.0935 \\
Herds & 0.2976 & 0.0227 & 0.0110 & 0.3432 \\
Residual & - & 0.3267 & 0.1662 & - \\
Variance ratio herd/sire & 4.60 & 3.49 & 3.68 & 3.67 \\
\hline
\end{tabular}

kept in all models. Other researchers have also included the duration of exposure in analysis of mastitis, finding a positive effect of DIM in liability to mastitis for a probit model (Heringstad et al., 2001) and number of cases of the disease for a zero inflated Poisson model (Rodrigues-Motta et al., 2007). The risk of mastitis is increased with DIM. However, cows with mastitis have a greater risk of being culled early in the lactation (expressing a reduced DIM). The "causal" effect of exposure (DIM) on mastitis cannot be identified with observational data. Heringstad et al. (2001) found an improvement in the likelihood of a model for mastitis when DIM was considered as a covariable compared with the same model excluding DIM.

Table 1 shows estimates of variance components for each of the models. In the Poisson model for NCM, the variance components apply to the $\log (\lambda)$ scale, whereas in the logit model for CM the components apply to a logit scale, and in the linear model estimates are in the scale of the response, either CM or NCM. Thus, estimates are not directly comparable between models. The ratios between herd and sire variances are more meaningful, being 4.60, 3.67, 3.68, and 3.49 in the Poisson, logit, and linear models for CM and for NCM, respectively (Table 1). Environmental factors are of major importance for mastitis incidence (Hogan and Smith, 2003). The "herd effect" represents several factors affecting disease, such as number and type of pathogens in the environment, management practices related to prevention of the disease, milking routines, etc. Although smaller than the estimate of between-herd variance, the between-sire variance was substantial, indicating the existence of genetic variation, which is necessary for genetic selection to be effective. RodriguesMotta et al. (2007) fitted an overdispersed Poisson model (including a residual in addition to sire and herd effects in the log-linear model) to the same data set using Bayesian methods. The ratio of the posterior mean estimates of the variance components was 4.28 , which is close to what was found in the present study (Table 1).

In a review, Heringstad et al. (2000) reported that most heritability estimates of CM obtained with linear models ranged from 0.02 to 0.03 , whereas heritability of liability to CM from threshold models was between 0.06 and 0.12 . In this study heritability estimates for the response variable $\mathrm{CM}$ were 0.07 and 0.11 for linear and logit model, respectively. In the Poisson model, $\lambda_{i j k l m}$ was a cow-specific parameter that ranged from 0.082 to 1.355 with a median of 0.252 and an average of 0.282 . Setting $\lambda$ to the median and to the mean respectively, yields $\hat{h}_{\eta}^{2}=0.06$ and 0.07 , respectively. Finally, the $\hat{h}^{2}$ for the linear model for NCM was 0.07.

\section{Prediction of Random Effects}

Prediction of sire effects for NCM ranged from -0.364 to 0.556 [Poisson model in the $\log (\lambda)$ scale] and from

Table 2. Correlations between a) random effect predictions (sire effects are given above the diagonal and herd effects below the diagonal) and b) expected values between models

\begin{tabular}{lcccc}
\hline & \multicolumn{4}{c}{ Model } \\
\cline { 2 - 5 } & Logit & Linear with CM & Poisson & Linear with NCM \\
\hline $\begin{array}{l}\text { a) Random effect predictions } \\
\text { Logit }\end{array}$ & - & 0.943 & 0.952 & 0.899 \\
$\quad$ Linear with CM & 0.999 & - & 0.894 & 0.955 \\
Poisson & 0.937 & 0.936 & - & 0.936 \\
Linear with NCM & 0.925 & 0.926 & 0.996 & - \\
b) Expected values & - & 0.998 & 0.921 & 0.921 \\
Logit & - & - & - & 0.923 \\
$\quad$ Linear with CM & - & - & - & - \\
$\quad$ Poisson & - & &
\end{tabular}


-0.158 to 0.233 (linear model in the NCM scale). Likewise, the corresponding predictions for $\mathrm{CM}$ ranged from -0.391 to 0.499 (logit model in the logit scale) and from -0.104 to 0.124 (linear model in the CM scale). An important question is to which extent prediction of sire effects agree across models. Table 2 a shows the correlation between predictions of sire effect of different models. Overall, the correlations were high (from 0.89 to 0.96 ) but were larger between the linear models (with different responses) followed by the correlation between nonlinear models (logit and Poisson). This suggests that the type of model, linear or nonlinear, had the greatest impact on the correlation. Considering a nonlinear model may re-order the sire predictions. Figure 1a shows a scatter plot of the predictions of sire effects from the Poisson and logit models.

Predictions for herd effects for NCM ranged from -0.926 to 1.281 [Poisson model in the $\log (\lambda)$ scale] and from -0.203 to 0.429 (linear model in the NCM scale). The corresponding predictions for $\mathrm{CM}$ ranged from -0.950 to 1.318 (logit model in the logit scale) and from -0.147 to 0.264 (linear models in the CM scale). The correlations between predictions of herd effects between the models were also high (Table 2a), especially between the models with the same response. Figure $1 \mathrm{~b}$ gives a scatter plot of the predictions of herd effects with logit and Poisson models. It should be noted that the association was stronger at smaller values of the predicted value, where both models are expected to match in outcome (zero mastitis cases). However, the association became weaker at greater values where, in the logit model, the outcome has collapsed to presence, whereas in the Poisson model there was variability between numbers of cases of the disease. For example, when only herds with predictions larger than zero in the logit scale were considered, the correlation was much less (0.80) than for the entire sample. A similar relation was observed between the herd predictions from the linear model with NCM and the predictors of logit. This suggests that considering the actual number of cases may be relevant for an adequate ranking of herds.

\section{Predictive Ability in Cross-Validation}

The correlation between the expected values of the models ranged from 0.921 to 0.998 , indicating general agreement. The greater correlations were between the models for counts (Poisson and linear model for NCM) and between the models for CM (logit and linear model for CM; Table 2b). All correlations between a model for NCM and a model for CM were close to 0.92 (Table $2 \mathrm{~b})$. The different trait definitions (CM or $\mathrm{NCM}$ ) were more important than the model itself from the point of view of the expected value. Within trait, logit and linear model for CM gave slightly more similar predictions $(\mathrm{r}=0.998)$ than Poisson and linear model for NCM response $(r=0.990)$. Figure 2 a shows the association between the estimated expectations for NCM from the Poisson and for CM from the logit model. Figure 2b shows the association between estimated expectations for NCM from the linear and Poisson models. Although they are highly correlated, Poisson expected estimates, $(\hat{\lambda})$, take larger values (with a maximum of 1.36 ) than

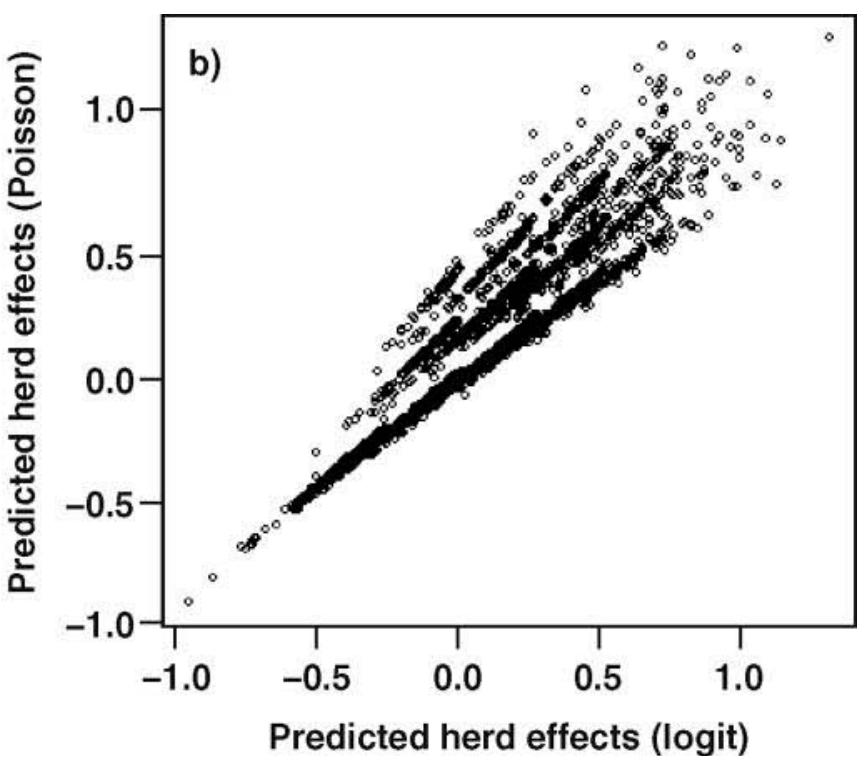

Figure 1. Scatter plot of predictions of sire (a) and herd (b) effects from the logit and Poisson models. 
the linear expected estimates (with a maximum of 0.76). The logit and linear model for CM yielded very similar CM estimated expectations, as shown in the plot (Figure 2c). Finally the association between the $\mathrm{NCM}$ and $\mathrm{CM}$ expectation estimates both from the linear model are shown in Figure 2d. The maximum of those estimates where similar $(0.76$ for $\mathrm{NCM}$ and 0.51 for $\mathrm{CM}$ ).

In cross-validation, the overall MSEP calculated with $\mathrm{NCM}$ as the variable to predict was similar for all the models, being 0.346 for Poisson, 0.345 for linear model for NCM, 0.350 for linear model for CM, and 0.351 for the logit. Table 3 shows the MSEP, with NCM as variable to predict, calculated separately for cows with different numbers of mastitis cases observed. Considering only cows with mastitis $(\mathrm{NCM} \geq 1$ and $\mathrm{CM}=1)$, models for NCM resulted in a smaller MSEP as well as in all the subsets with more than 1 case of mastitis (i.e., that yield a prediction closer to the observed values). On the other hand, models for CM, logit and linear with binary response showed a better predictive ability when predicting zeros (healthy cows) than the
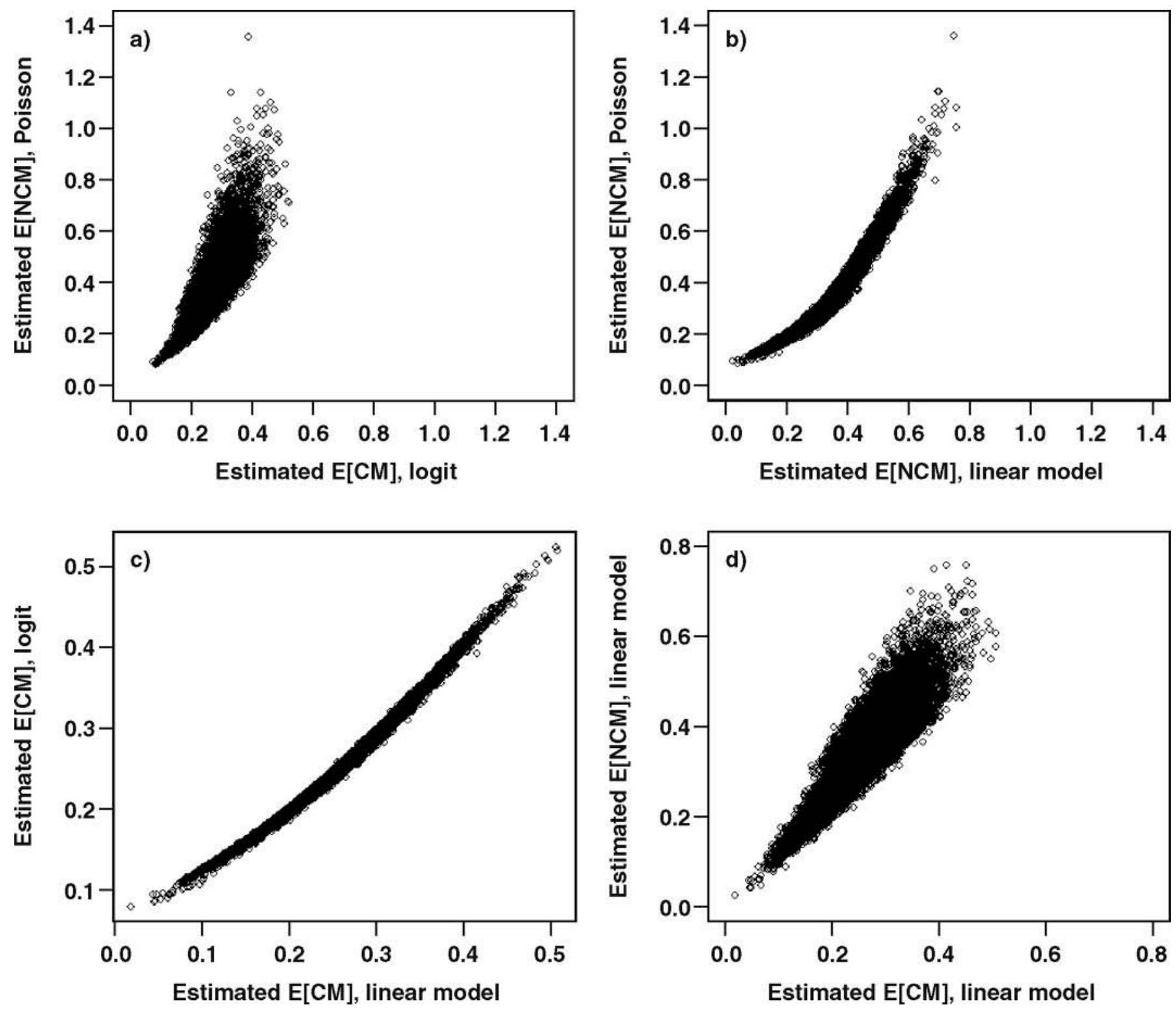

Figure 2. Scatter plot of estimated expectations for cows from the cross-validation of (a) number of clinical mastitis cases (NCM) with the Poisson model and clinical mastitis (CM) with the logit model; (b) NCM with the Poisson model and NCM with the linear model; (c) CM with the logit and CM with the linear model; and (d) NCM with the linear and CM with the linear model. 
Table 3. Mean squared error of prediction for $0, \geq 1,1,2$, and $\geq 3$ observed cases of clinical mastitis (CM) for Poisson, logit, and linear models

\begin{tabular}{lccccc}
\hline & \multicolumn{5}{c}{ Mastitis cases observed } \\
\cline { 2 - 6 } Model & 0 (healthy) & $\geq 1$ (diseased) & 1 & 2 & $\geq 3$ \\
\hline Poisson & 0.085 & 1.206 & 0.499 & 2.823 & 7.143 \\
Linear NCM & 0.090 & 1.185 & 0.480 & 2.800 & 7.110 \\
Linear CM & 0.056 & 1.319 & 0.573 & 3.046 & 7.511 \\
Logit & 0.053 & 1.333 & 0.583 & 3.072 & 7.555 \\
\hline
\end{tabular}

${ }^{1} \mathrm{NCM}=$ number of $\mathrm{CM}$ cases.

models for NCM. Within the models for NCM, Poisson predicted more accurately the healthy cows but less accurately the count of cases than the linear model, although they were similar and the difference could be due to sampling. Within the models for binary response (CM) linear model better predicted the mastitic cows and worse the healthy cows. However, those models yielded very similar results, and the difference between them could also be due to sampling.
Figure 3 shows the distribution of the estimated expected NCM for the 4 models. The distribution is presented by observed NCM, Figure 3a corresponds to cows with $0 \mathrm{CM}$ and Figure $3 \mathrm{~b}$ corresponds to cows with 3 or more cases of mastitis. The distributions for cows with 1 and 2 mastitis events in the lactation were in between the extreme cases showed in Figure 3. For the Poisson model, the distribution always had a thicker right tail, especially for cows that had mastitis,

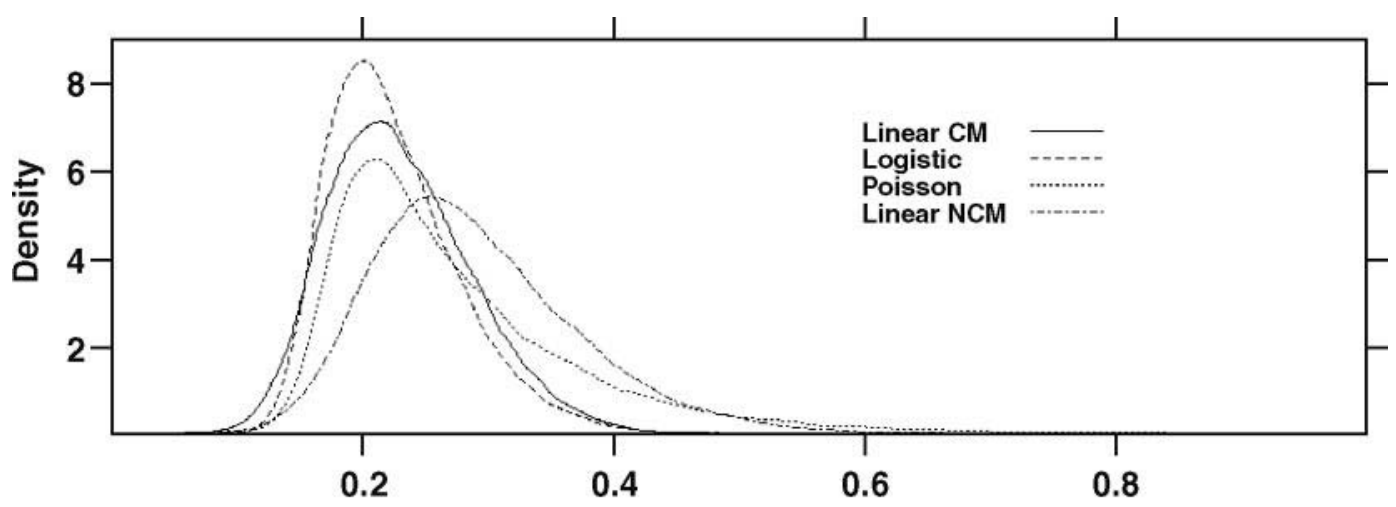

Estimated $\mathrm{E}[\mathrm{NCM}]$ when the observed $\mathrm{NCM}$ is 0 (NCM)

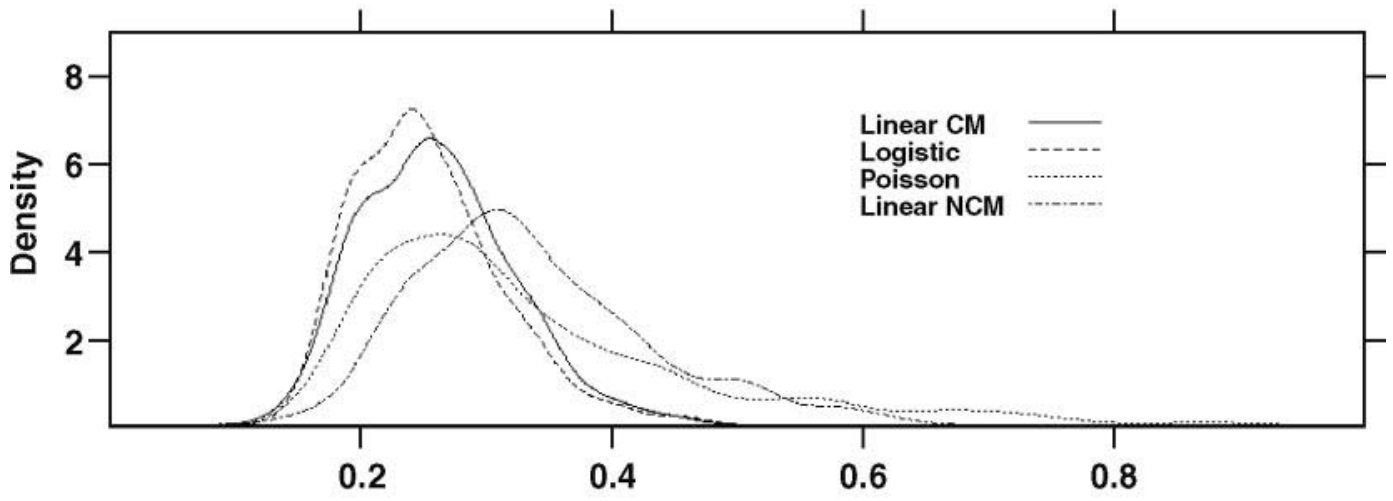

Estimated $\mathrm{E}[\mathrm{NCM}]$ when the observed NCM is 3 or more (NCM)

Figure 3. Predicted number of cases of clinical mastitis (NCM) for cows that had an observation for the number of mastitis cases of 0 or healthy (a), and 3 or more (b). 
which is why the predicted number of events in sick cows was more accurate with a Poisson model, whereas the logit model had a smaller MSEP when addressing healthy cows. The linear model with NCM response had an overall increased estimated expected NCM. The distribution shows why that model performed worse for prediction of healthy cows. The distribution for the Poisson model was more similar to the other 2 distributions and differed only in the tail. The distribution of predictions from the linear model was very similar to that of the logit specification.

\section{CONCLUSIONS}

Predictions of sire effects from Poisson, logit, and linear models were highly correlated. However, a lesser correlation was observed between models that had a different response. The cross-validation study suggested similar overall predictive performance for all the models tested. Trait definition (CM or NCM) made a larger difference in the MSEP than the model used. The Poisson and linear models for NCM behave better to predict mastitic cows, as well as for predicting the specific number of mastitis cases $(1,2,3$, or more). Linear model with NCM response was the best model predicting mastitic cows and the worst one predicting healthy cows, although the difference with Poisson was small and could be due to sampling. Although the NCM expected estimates were highly correlated, Poisson estimates showed a larger range than linear model estimates. Predictions for healthy cows were closer to the observed value with the logit model, followed closely by the linear model with CM response. The logit model showed the most extreme results, being the best model for predicting healthy cows and the worst model for prediction of sick cows.

\section{ACKNOWLEDGMENTS}

Access to the data was given by the Norwegian Dairy Herd Recording System (Ås, Norway) and the Norwegian Cattle Health Service (Ås, Norway) in agreement number 004.2005. Research was funded by grants USDA 2003-35205-12833, NSF DEB-0089742, and NSF DMS 0443771. Support by the Wisconsin Agriculture Experiment Station and by the Babcock Institute for Dairy Research and Development is acknowledged. Valuable suggestions from 2 referees are greatly appreciated.

\section{REFERENCES}

Agresti, A. 2002. Categorical Data Analysis. 2nd ed. Wiley, New York, NY.
Bates, D., and D. Sarkar. 2005. lme4: Linear mixed-effects model using S4 classes. http://www.r-project.org/ Accessed Nov. 2008.

Bulmer, M. G. 1980. The Mathematical Theory of Quantitative Genetics. Oxford, New York, NY.

Caraviello, D. Z., K. A. Weigel, and D. Gianola. 2004. Comparison between a Weibull proportional hazards model and linear model for predicting the genetic merit of US Jersey sires for daughter longevity. J. Dairy Sci. 87:1469-1476.

De Haas, Y., H. W. Barkema, and R. F. Veerkamp. 2002. Genetic parameters of pathogen-specific incidence of clinical mastitis in dairy cows. Anim. Sci. 74:233-242.

Doran, H., D. Bates, P. Bliese, and M. Dowling. 2007. Estimating the multilevel Rasch model with the lme4 Package. J. Stat. Softw. 20:1-18.

Foulley, J. L., D. Gianola, and S. Im. 1987. Genetic evaluation for traits distributed as Poisson binomial with reference to reproductive characters. Theor. Appl. Genet. 73:870-877.

Gasqui, P., O. Pons, and J. B. Coulon. 2000. An individual modeling tool for consecutive clinical mastitis during the same lactation in dairy cows: A method based on a survival model. Vet. Res. 31:583-602.

Gianola, D., and R. L. Fernando. 1986. Bayesian methods in animal breeding theory. J. Anim. Sci. 63:217-244.

Gianola, D., and J. L. Foulley. 1983. Sire evaluation for ordered categorical data with a threshold model. Genet. Sel. Evol. 15:201-224.

Harville, D. A., and T. P. Callanan. 1989. Computational aspects of likelihood-based inference for variance components. Pages 136-176 in Statistical Methods for Genetic Improvement of Livestock. D. Gianola and K. Hammond, ed. Springer-Verlag, Berlin, Germany.

Henderson, C. R. 1973. Sire evaluation and genetic trends. Pages 1041 in Proc. Anim. Breed. Genet. Symp., Blacksburg, VA. Am. Soc. Anim. Sci., Champaign, IL.

Henderson, C. R. 1975. Best linear unbiased estimation and prediction under a selection model. Biometrics 31:423-447.

Henderson, C. R. 1976. A simple method for computing the inverse of a numerator relationship matrix used in prediction of breeding values. Biometrics 32:69-83.

Heringstad, B., Y. M. Chang, D. Gianola, and G. Klemetsdal. 2003. Genetic analysis of longitudinal trajectory of clinical mastitis in first-lactation Norwegian cattle. J. Dairy Sci. 86:2676-2683.

Heringstad, B., Y. M. Chang, D. Gianola, and G. Klemetsdal. 2004. Multivariate threshold model analysis of clinical mastitis in multiparous Norwegian dairy cattle. J. Dairy Sci. 87:3038-3046.

Heringstad, B., G. Klemetsdal, and J. Ruane. 2000. Selection for mastitis resistance in dairy cattle - A review with focus on the situation in the Nordic countries. Livest. Prod. Sci. 64:95-106.

Heringstad, B., R. Rekaya, D. Gianola, G. Klemetsdal, and K. A. Weigel. 2001. Bayesian analysis of liability of clinical mastitis in Norwegian cattle with a threshold model: effects of data sampling method and model specification. J. Dairy Sci. 84:2337-2346.

Hogan, J., and K. L. Smith. 2003. Coliform mastitis. Vet. Res. 34:507-519.

McCullagh, P., and J. A. Nelder. 1989. Generalized Linear Models. 2nd ed. Chapman and Hall, London, UK.

National Mastitis Council. 2004. The value and use of DHI somatic cell count. http://www.nmconline.org/dhiscc.htm Accessed December 2007.

Quaas, R. L., and E. J. Pollak. 1980. Mixed model methodology for farm and ranch beef cattle testing programs. J. Anim. Sci. 51:1277-1287.

R Development Core Team. 2008. R: A language and environment for statistical computing. R Foundation for Statistical Computing, Vienna, Austria. http://www.r-project.org Accessed Nov. 2008.

Rekaya, R., S. L. Rodriguez-Zas, D. Gianola, and G. E. Shook. 1998. Test-day models for longitudinal binary responses: An application to mastitis in Holsteins. Page 44 in Book of Abstracts, 49th Annu. Meet. EAAP, Warsaw, Poland. Wageningen Pers, Wageningen, the Netherlands

Rodrigues-Motta, M., D. Gianola, B. Heringstad, G. J. M. Rosa, and Y. M. Chang. 2007. A zero-inflated Poisson model for genetic 
analysis of number of mastitis cases in Norwegian Red cows. J. Dairy Sci. 90:5306-5315.

Rupp, R., and D. Boichard. 1999. Genetic parameters for clinical mastitis, somatic cell score, production, udder type traits, and milking ease in first lactation Holsteins. J. Dairy Sci. 82:21982204.

Sæbø, S., T. Almøy, B. Heringstad, G. Klemetsdal, and A. H. Aastveit. 2005. Genetic evaluation of mastitis resistance using a first passage time model for Wiener processes for analyses of time to first treatment. J. Dairy Sci. 88:834-841.
Schukken, Y. H., F. J. Grommers, D. Van de Geer, H. N. Erb, and A. Brand. 1990. Risk factors for clinical mastitis in herds with a low bulk milk somatic cell count. 1. Data and risk factors for all cases. J. Dairy Sci. 73:3463-3471.

Vazquez, A. I. 2007. Analysis of number of episodes of clinical mastitis in Norwegian Red and Holstein cows with Poisson and categorical data mixed models. MS thesis. University of Wisconsin, Madison. 